\title{
MicroRNA-145 inhibits lung cancer cell metastasis
}

\author{
DONG-JIN LING ${ }^{1,2^{*}}$, ZHONG-SHU CHEN $^{1,2^{*}}$, YANG-DE ZHANG ${ }^{1}$, QIAN-DE LIAO ${ }^{3}$, \\ JIAN-XIONG FENG ${ }^{2}$, XUE-YU ZHANG ${ }^{2}$ and TIAN-SHENG SHI ${ }^{2}$
}

\begin{abstract}
${ }^{1}$ Department of General Surgery, Ministry of Health Hepatobiliary and Enteric Surgery Center, Xiangya School of Medicine, Central South University, Changsha, Hunan 410008; ${ }^{2}$ Department of Thoracic Surgery, Jiangxi Provincial Chest Hospital, Nanchang, Jiangxi 330006; ${ }^{3}$ Department of Orthopaedics, Xiangya Hospital of Central South University, Changsha, Hunan 410008, P.R. China
\end{abstract}

Received April 1, 2014; Accepted October 31, 2014

DOI: $10.3892 / \mathrm{mmr} .2014 .3036$

\begin{abstract}
Previous studies have identified a variety of microRNAs (miRNAs) that have important roles in cancer progression, particularly in tumor invasion and metastasis. Downregulation of miR-145 was reported to occur in various types of human cancer; however, the role of miR-145 in lung cancer metastasis and its potential mechanisms of action remain to be elucidated. The present study aimed to investigate the effects of miR-145 on metastasis and epithelial-mesenchymal transition (EMT) in A549 human lung adenocarcinoma cells. In addition, the underlying mechanisms by which miR-145 regulates EMT were examined. The miR-145 mimic was transfected into A549 cells; cell invasion and adhesion assays were then performed in order to investigate cell metastasis, and western blot analysis was used to examine the expression of EMT markers. In order to further examine the underlying mechanisms by which miR-145 regulates EMT, a luciferase reporter assay was performed to determine whether miR-145 targeted Oct 4 . In addition, the expression of Wnt $3 \mathrm{a}$ and $\beta$-catenin in A549 cells was measured following transfection with small hairpin RNA-Oct4. To the best of our knowledge, the results of the present study demonstrated for the first time, that miR-145 inhibited lung cancer cell metastasis and EMT via targeting the Oct4 mediated $\mathrm{Wnt} / \beta$-catenin signaling pathway.
\end{abstract}

Correspondence to: Professor Yang-De Zhang, Department of General Surgery, Ministry of Health Hepatobiliary and Enteric Surgery Center, Xiangya School of Medicine, Central South University, 87 Xiangya Road, Changsha, Hunan 410008, P.R. China E-mail: ydzhangxy@163.com

*Contributed equally

Key words: microRNA-145, epithelial-mesenchymal transition, lung cancer, octamer-binding transcription factor 4, wingless-type MMTV integration site family $/ \beta$-catenin signaling

\section{Introduction}

Lung cancer is the most prevalent type of cancer, accounting for $>26 \%$ of all cancer-associated mortalities (1). Mortality among lung cancer patients is primarily caused by metastasis; therefore, it is essential to elucidate the mechanisms involved in lung cancer metastasis in order to determine novel therapeutic targets. Cancer metastasis is a complex process in which cancer cells migrate from the primary tumor and invade into the surrounding tissues (2). Epithelial-mesenchymal transition (EMT) provides a novel basis for understanding the progression and metastasis of cancer (3-5). During EMT, polarized epithelial cells lose their epithelial characteristics and are converted to motile mesenchymal-like cells (6).

The number of studies regarding the critical roles of micro (mi)RNAs in EMT is increasing (7). miRNAs are a class of small noncoding RNAs that bind to areas in the 3'-untranslated region (UTR) of target mRNAs (8), resulting in either mRNA degradation or translational repression (9). Previous studies have identified a variety of miRNAs that have important roles in cancer progression, particularly in tumor invasion and metastasis (10).

Downregulation of miR-145 was reported to occur in various types of human cancer (11-13), in which it exerts its function in a cell-specific manner (14-16). Yin et al (17) demonstrated that miR-145 suppressed human lung adenocarcinoma-initiating cell proliferation, which resulted in the inhibition of lung cancer development. However, the role of miR-145 in lung cancer metastasis and its potential mechanisms of action remain to be elucidated. The present study aimed to investigate the effects of miR-145 on metastasis and EMT in A549 human lung adenocarcinoma cells. In addition, the underlying mechanisms by which miR-145 regulates EMT were examined.

\section{Materials and methods}

Reagents and antibodies. The pRL-TK Renilla luciferase reporter vector, pmirGLO luciferase reporter vector and Dual-Luciferase Reporter Assay system were purchased from Promega Corporation (Madison, WI, USA). The following antibodies: Rabbit polyclonal Oct4 (sc-9081), mouse monoclonal Wnt3a (sc-136163), rabbit polyclonal $\beta$-catenin 
(sc-7199), mouse monoclonal GAPDH (sc-365062), horseradish peroxidase (HRP)-conjugated goat anti-mouse immunoglobulin (IgG) and HRP-conjugated goat anti-rabbit IgG were purchased from Santa Cruz Biotechnology, Inc. (Dallas, TX, USA). Rabbit polyclonal vimentin antibody (ab92547) was obtained from Abcam (Cambridge, MA, USA). Rabbit polyclonal N-cadherin (SAB, 21474), rabbit polyclonal E-cadherin (SAB , 21473) and rabbit polyclonal pan-cytokeratin (SAB, 23032) were obtained from Signalway Antibody Inc. (College Park, MD, USA). The miR-145 mimic, miR-145 inhibitor and miR-145 negative control (NC) were synthesized by Biomics Biotechnology Co., Ltd (Jiangsu, China).

Cell culture. Human lung cancer cell lines, SPC-A-1, A549, LTEP-a-2, SK-LU-1 and GLC-82, as well as the normal human bronchial epithelial (HBE) cell line were purchased from the American Type Culture Collection (Manassas, VA, USA) and maintained in Dulbecco's modified Eagle's medium (Gibco-BRL, Grand Island, NY, USA) containing 10\% fetal bovine serum (FBS; Gbico-BRL) at $37^{\circ} \mathrm{C}$ in a humidified atmosphere with $5 \% \mathrm{CO}_{2}$.

Tissues. Tumor and adjacent normal lung tissue specimens were collected from 10 patients with non-small-cell lung cancer in Jiangxi Provincial Chest Hospital (Jiangxi, China). This study was approved by the Ethics Committee of Jiangxi Provincial Chest Hospital. All patients provided written informed consent in compliance with the code of ethics of the Declaration of Helsinki. None of the patients enrolled in the present study had received chemotherapy or radiotherapy prior to surgery. The tumor and adjacent normal tissue samples were frozen in liquid nitrogen immediately following surgery and stored at $-80^{\circ} \mathrm{C}$ until further use.

Reverse transcription-quantitative polymerase chain reaction $(R T-q P C R)$. Total RNA was extracted from tissue samples or cells using TRIzol ${ }^{\circledR}$ (Invitrogen Life Technologies, Carlsbad, CA, USA), miR-145 was isolated using an miRNeasy Mini kit (Qiagen, Limburg, Netherlands) according to the manufacturer's instructions. Approximately $5 \mu \mathrm{g}$ RNA was able to be isolated from $1 \mathrm{mg}$ tissue or $1 \times 10^{6}$ cells. Complementary DNA (cDNA) was synthesized using a First Strand cDNA Synthesis kit (Fermentas, Vilnius, Lithuania) using $1 \mu \mathrm{g}$ RNA template. The primers were as follows: miR-145 forward, 5'-GTCCAGTTTTCCCAGGAATCCCT-3' and reverse, 5'-TGGTGTCGTGGAGTCG-3'; U6 forward, 5'-GCTTCGGCAGCACATATACTAAAAT-3' and reverse, 5'-CGCTTCACGAATTTGCGTGTCAT-3'. qPCR was performed using a SYBR-Green PCR kit (Applied Biosystems, Foster City, CA, USA) in a 7300 Sequence Detection system (Applied Biosystems). The PCR conditions consisted of $5 \mathrm{~min}$ of initial denaturation at $95^{\circ} \mathrm{C}, 40$ cycles of denaturation at $95^{\circ} \mathrm{C}$ for $10 \mathrm{sec}$, annealing at $60^{\circ} \mathrm{C}$ for $20 \mathrm{sec}$ and elongation at $72^{\circ} \mathrm{C}$ for $10 \mathrm{sec}$. The relative quantities of each mRNA were calculated using the comparative CT methods.

Western blot analysis. Tissues and cells were lysed using a total protein extraction kit (Promab, Inc., Hunan, China). Protein concentrations were determined using the bicinchoninic acid kit (Beyotime, Shanghai, China). A total of $50 \mu \mathrm{g}$ protein was separated using 10\% SDS-PAGE and blotted onto nitrocellulose membranes (Millipore, Billerica, MA, USA). Following blocking in $5 \%$ non-fat milk overnight at $4^{\circ} \mathrm{C}$, the membranes were incubated with the primary antibodies for $1 \mathrm{~h}$ at room temperature, followed by incubation with the secondary antibodies for $1 \mathrm{~h}$ at room temperature. Detection was performed using an enhanced chemiluminescence kit (Pierce Biotechnolgy, Inc., Rockford, IL, USA). GAPDH was used as a loading control.

Transfection. Transfection was performed using Lipofectamine $^{\mathrm{TM}} 2000$ (Invitrogen Life Technologies) according to the manufacturer's instructions. Briefly, $4 \times 10^{4}$ cells were seeded into six-well plates and incubated at $37^{\circ} \mathrm{C}$ with $5 \% \mathrm{CO}_{2}$ for $24 \mathrm{~h}$. Prior to transfection, $5 \mu \mathrm{l}$ Lipofectamine ${ }^{\mathrm{TM}} 2000$ was diluted in $250 \mu \mathrm{l} \mathrm{Opti-MEM}{ }^{\circledR} \mathrm{I}$ (Gibco-BRL) and incubated for $5 \mathrm{~min}$. The miR-145 mimic or inhibitor was diluted in $250 \mu \mathrm{l}$ Opti-MEM ${ }^{\circledR}$ I to a final concentration of $2 \mu \mathrm{M}$. The two solutions were mixed gently and incubated at room temperature for $20 \mathrm{~min}$. The lipid-DNA complexes were added to each well and cells were incubated at $37^{\circ} \mathrm{C}$. Sequences for the miR-145 mimic were: Sense, 5'-GUCCAGUUUUCCCAGGAAUCCCU-3' and antisense, 5'-GGAUUCCUG GGAAAACUGGACUU-3'. Sequences for the miR-145 inhibitor were: antisense, 5'-AGGGAUUCCUGGGAAAACUG GAC-3'. Following 6 h, cells were incubated with fresh medium and maintained in the cultures for a minimum of $48 \mathrm{~h}$; cells were then harvested for analysis. To knockdown Oct4 in A549 cells, small hairpin(sh)RNAs for Oct4 were constructed and the DNA sequences were cloned into pGPU6/GFP/ Neo vectors (Invitrogen Life Technologies). The most effective shRNA sequences were: sense, 5'-CACCGGGTAGGT TATTTCTAGAAGTTTCAAGAGAACTTCTAGAAATAACC TACCCTTTTTTG-3' and antisense, 5'-GATCCAAAAAAG GGTAGGTTATTTCTAGAAGTTCTCTTGAAACTTCTAG TAGAAATAACCTACCC-3'. The knockdown of Oct4 in A549 cells was generated by transfection with $2 \mu \mathrm{g}$ shRNA-Oct4.

Luciferase assay. Reporter plasmids containing 3'UTR Oct4 (pmirGLO-Oct4) were co-transfected with miR-NC or miR-145 mimic into A549 cells. pRL-TK Renilla luciferase reporter vector was used as an internal control in each assay. Firefly and Renilla luciferase activities were measured using the Dual-Luciferase Reporter Assay system. Results were expressed as firefly luciferase activity normalized to Renilla luciferase activity.

Transwell-matrigel invasion assay. Cell invasion assays were performed using Transwell inserts pre-coated with

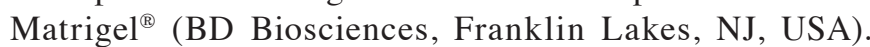
A549 cells were harvested and resuspended in serum-free medium in order to obtain a density of $5 \times 10^{4}$ cells $/ \mathrm{ml}$. A total of $1 \mathrm{ml}$ cell suspension was added to the upper chambers, the lower chamber was filled with $1 \mathrm{ml}$ cell medium containing $10 \%$ FBS. Following incubation at $37^{\circ} \mathrm{C}$ for $12 \mathrm{~h}$, non-invaded cells were removed using a cotton swab, the invaded cells were fixed using 95\% ethanol for $15 \mathrm{~min}$ and then stained with hematoxylin for $10 \mathrm{~min}$. The invaded cells were counted under a light microscope (TS100; Nikon, Tokyo, Japan). 
A

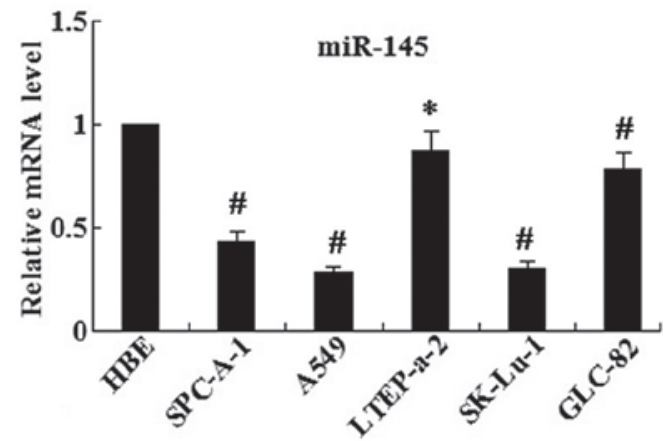

B

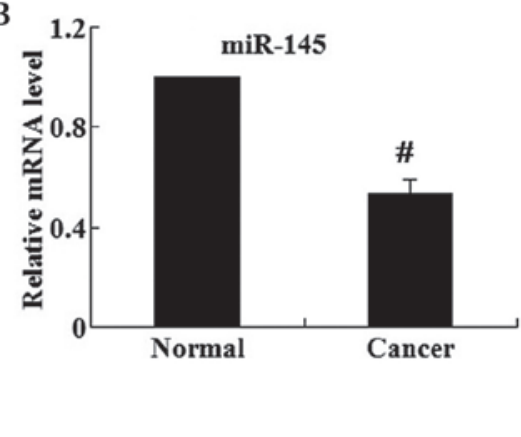

Figure 1. Expression of miR-145 in human lung cancer tissues and cell lines was determined using reverse transcription-quantitative polymerase chain reaction. (A) Relative mRNA expression levels of miR-145 in human lung cancer cell lines as well as in the normal HBE cells. (B) Relative mRNA expression levels of miR-145 in human lung cancer tissues and adjacent normal tissues. Values are presented as the mean \pm standard deviation. ${ }^{*} \mathrm{P}<0.05$ and ${ }^{\#} \mathrm{P}<0.01$ vs. the normal group. miR, microRNA; HBE, human bronchial epithelial.
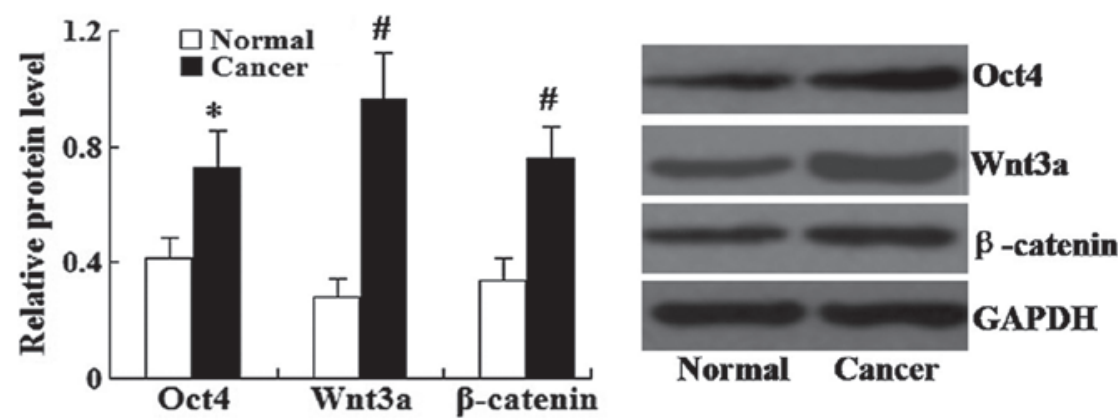

Figure 2. Western blot analysis was used to detect protein expression levels of Oct4, Wnt3a and $\beta$-catenin in human lung cancer tissues and adjacent normal tissues. Values are presented as the mean \pm standard deviation. ${ }^{*} \mathrm{P}<0.05$ and ${ }^{~} \mathrm{P}<0.01$ vs. the normal group.

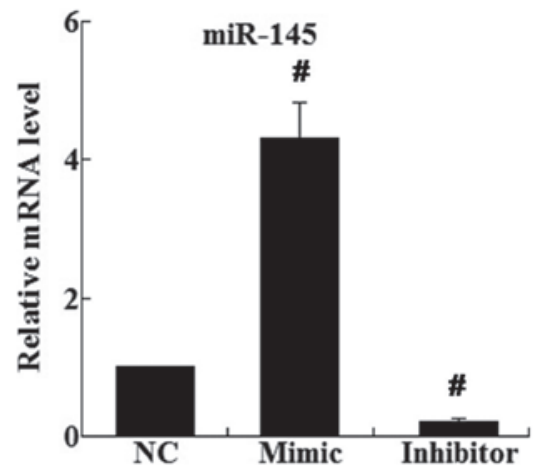

Figure 3. Relative mRNA expression levels of miR-145 in A549 cells following transfection with the miR-145 mimic, miR-145 inhibitor and miR-NC. Values are presented as the mean \pm standard deviation. ${ }^{\#} \mathrm{P}<0.01$ vs. the $\mathrm{NC}$ group. miR, micro RNA; NC, negative control.

Adhesion assay. Fibronectin (Sigma-Aldrich, St. Louis, MO, USA) was used to coat 96 -well plates and the plates were left for $2 \mathrm{~h}$. The wells were then blocked with $1 \%$ bovine serum albumin in phosphate-buffered saline (PBS; Maixin, Fuzhou, China) for a further $2 \mathrm{~h}$. Cells were suspended at a final concentration of $3 \times 10^{5}$ cells $/ \mathrm{ml}$ in serum-free medium prior to seeding into the wells. Following incubation for $2 \mathrm{~h}$, the wells were washed with PBS to remove non-adherent cells, and then fixed in paraformaldehyde (Xinchenghuagong, Inc., Guangzhou, China). The number of adherent cells was determined using the colorimetric
MTT assay (MTT reagent; Sigma-Aldrich) according to the manufacturer's instructions.

Statistical analysis. Values are presented as the mean \pm standard deviation of at least three independent experiments. Statistical analysis was performed using SPSS 19.0 statistical software (IBM, Armonk, NY, USA). Student's t-test was used to evaluate differences between groups. $\mathrm{P}<0.05$ was considered to indicate a statistically significant difference between values.

\section{Results}

miR-145 is decreased in human lung cancer cell lines and human lung cancer tissues. The relative mRNA levels of miR-145 in a series of human lung cancer cell lines, SPC-A-1, A549, LTEP-a-2, SK-LU-1 and GLC-82, as well as the normal human bronchial epithelial (HBE) cells were examined using RT-qPCR. The results demonstrated that the relative mRNA expression levels of miR-145 in human lung cancer cell lines were significantly reduced compared with those of the normal HBE cells (all $\mathrm{P}<0.05$ except LTEP-a-2 $\mathrm{P}<0.01$ ) (Fig. 1A). In addition, A549 cells demonstrated the lowest mRNA expression levels of miR-145. Furthermore, the expression levels of miR-145 in human lung cancer and adjacent normal tissues were examined (Fig. 1B); it was revealed that the expression levels of miR-145 were significantly decreased in the lung cancer tissues compared with those in the adjacent normal tissues $(\mathrm{P}<0.01)$. 


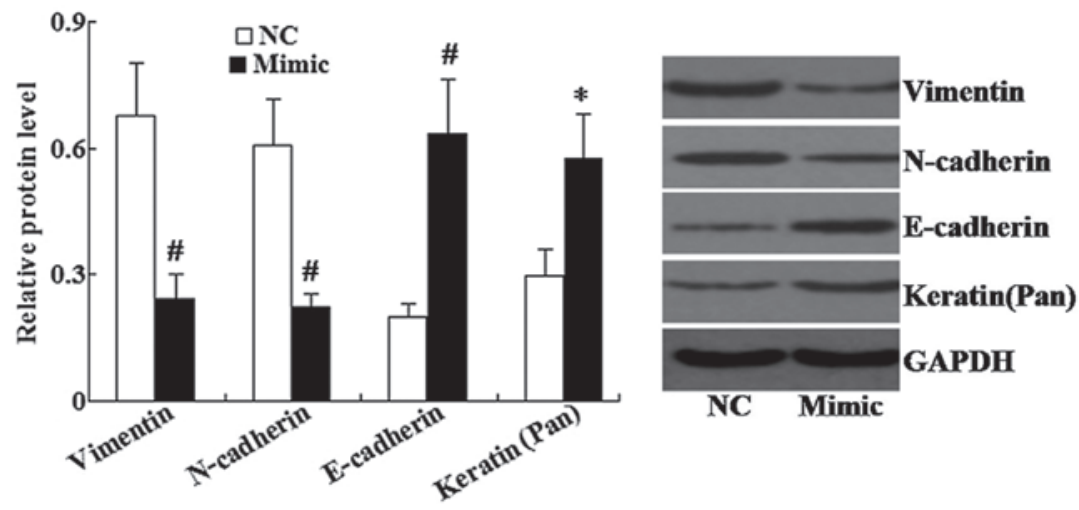

Figure 4. Western blot analysis of the protein expression levels of mesenchymal markers Vimentin and N-cadherin, as well as epithelial markers E-cadherin and Pan-Keratin in A549 cells following transfection with the miR-145 mimic and miR-NC. Values are presented as the mean \pm standard deviation. ${ }^{*} \mathrm{P}<0.05$ and ${ }^{\#} \mathrm{P}<0.01$ vs. the $\mathrm{NC}$ group. $\mathrm{NC}$, negative control; miR, microRNA.
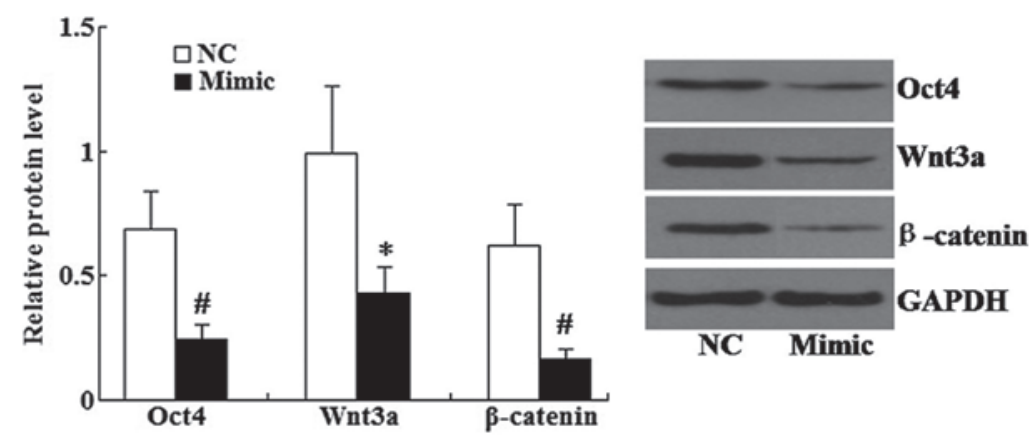

Figure 5. Western blot analysis of the protein expression levels of Oct4, Wnt3a and $\beta$-catenin in A549 cells following transfection with the miR-145 mimic and miR-NC. Values are presented as the mean \pm standard deviation. ${ }^{~} \mathrm{P}<0.05$ and ${ }^{\prime \prime} \mathrm{P}<0.01$ vs. the $\mathrm{NC}$ group. $\mathrm{NC}$, negative control; miR, microRNA.
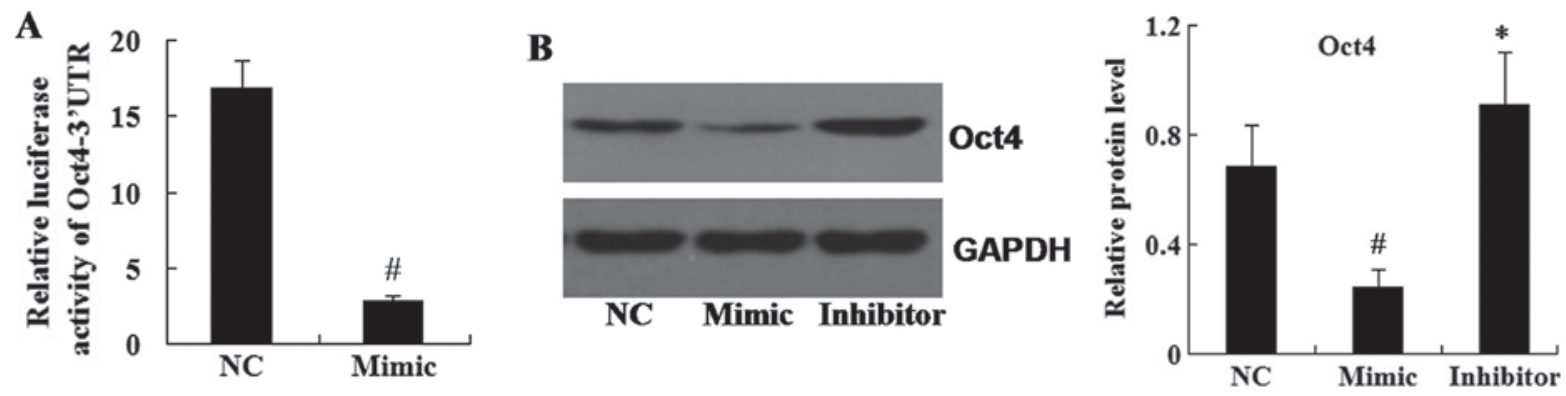

Figure 6. Oct4 is a direct target of miR-145. (A) Luciferase assays of pmirGLO-Oct4-3'UTR reporter activity co-transfected with the miR-NC and the miR-145 mimic. (B) Western blot analysis of the protein expression levels of Oct4 in A549 cells following transfection with the miR-145 mimic and the miR-145 inhibitor. Values are presented as the mean \pm standard deviation. " $\mathrm{P}<0.05$ and ${ }^{\#} \mathrm{P}<0.01$ vs. the $\mathrm{NC}$ group. NC, negative control; miR, microRNA; UTR, untranslated region.

Relative protein expression of Oct4, Wnt 3 a and $\beta$-catenin is increased in human lung cancer tissues. Western blot analysis was used to determine the relative protein expression levels of Oct4, Wnt3a and $\beta$-catenin in human lung cancer tissues. As shown in Fig. 2, the relative protein levels of Oct4, Wnt3a and $\beta$-catenin in the lung cancer tissues were all significantly increased compared with those in the adjacent normal tissues ( $\mathrm{P}<0.05$ for Oct- 4 and $\mathrm{P}<0.01$ for Wnt $3 \mathrm{a}$ and $\beta$-catenin).

Restoration of miR-145 inhibits the metastasis and EMT of A549 cells. miR-145 mimic and inhibitor were transfected into A549 cells. As shown in Fig. 3, RT-qPCR demonstrated that the relative mRNA expression of miR-145 in the mimic group was significantly increased 4.31-fold compared with that of the control group $(\mathrm{P}<0.01)$; however, miR-145 in the inhibitor group was significantly decreased to $22.01 \%$ of the control group $(\mathrm{P}<0.01)$ (Fig. 3).

Following transfection of the miR-145 mimic into A549 cells, cell invasion and adhesion assays were performed in order to determine the metastasis and EMT of the transfected cells. The results of the invasion assay revealed that the miR-145 mimic significantly inhibited cell invasion; the number of invaded cells in the miR-145 mimic group was markedly decreased compared with that of the control group $(21 \pm 4$ and $36 \pm 6$, respectively; $\mathrm{P}<0.05)$. Cell adhesion assays demonstrated that following transfection with the 


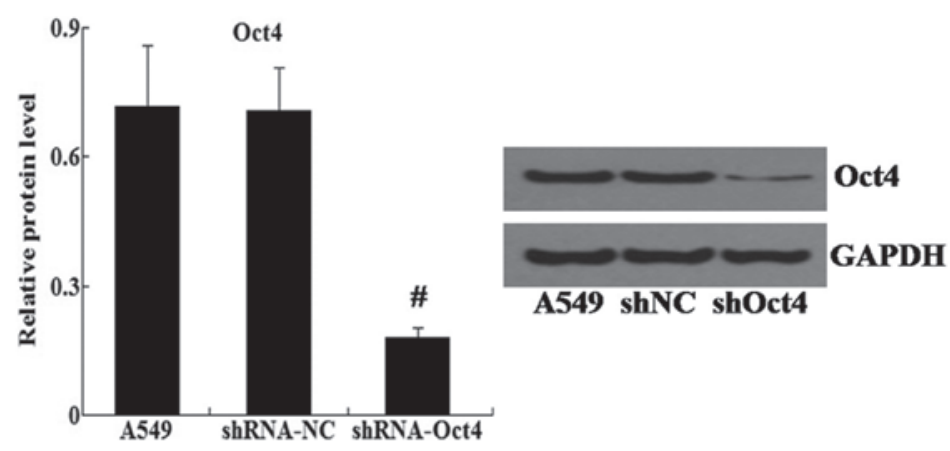

Figure 7. Western blot analysis of the protein expression levels of Oct4 in A549 cells following transfection with shRNA-NC and shRNA-Oct4. ${ }^{\text {"P }}<0.01$ vs. NC group. NC, negative control; sh/shRNA, small hairpin RNA.

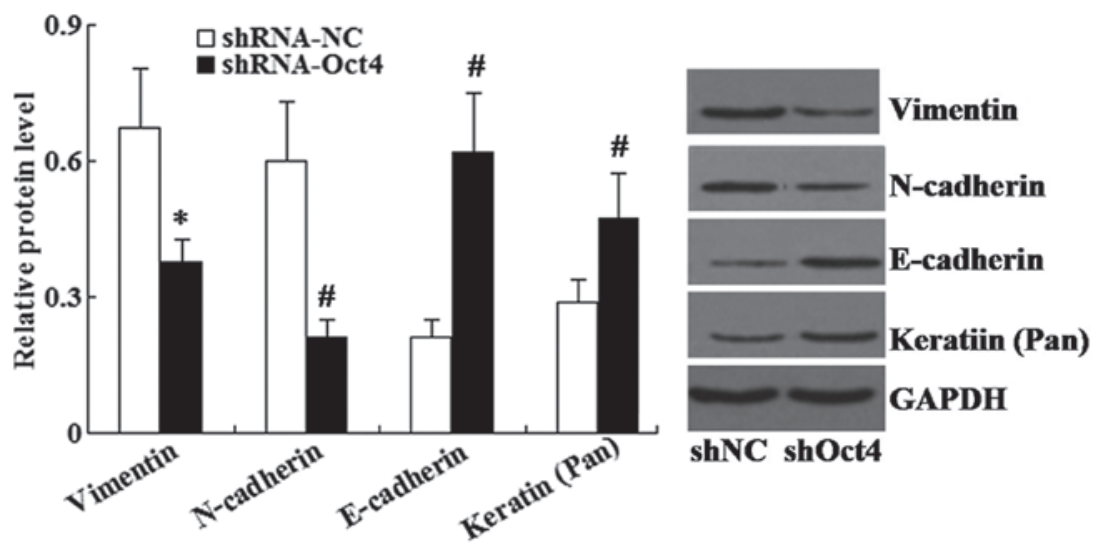

Figure 8. Western blot analysis of the protein expression levels of mesenchymal markers Vimentin and N-cadherin, as well as epithelial markers E-cadherin and Pan-Keratin in A549 cells following transfection with shRNA-NC and shRNA-Oct4. Values are presented as the mean \pm standard deviation. ${ }^{*}<<0.05$ and ${ }^{\text {"P }}<0.01$ vs. the NC group. NC, negative control; sh/shRNA, small hairpin RNA.

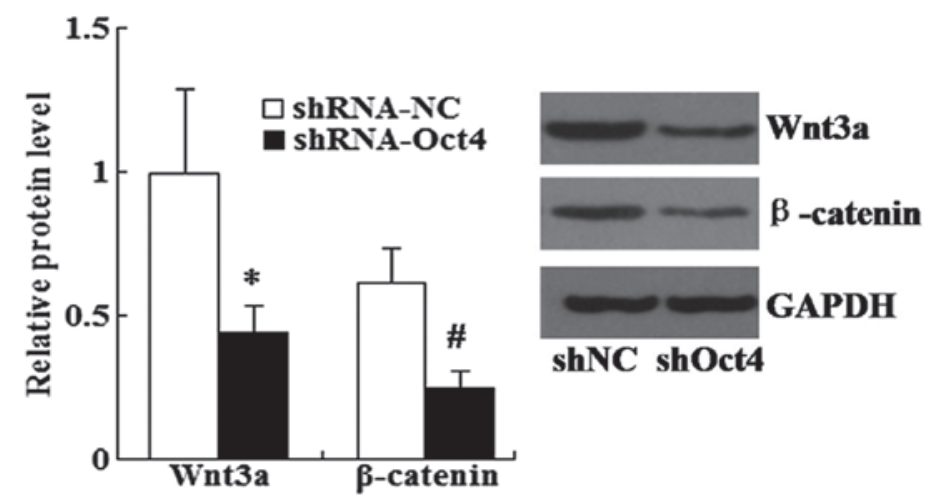

Figure 9. Western blot analysis of the protein expression levels of Wnt3a and $\beta$-catenin in A549 cells following transfection with shRNA-NC and shRNA-Oct4 Values are presented as the mean \pm standard deviation. " $\mathrm{P}<0.05$ and ${ }^{~} \mathrm{P}<0.01$ vs. the NC group. NC, negative control; sh/shRNA, small hairpin RNA.

miR-145 mimic, the adhesion activity of A549 cells was reduced to $51.01 \%$ of the untransfected control $(\mathrm{P}<0.05$, data not shown).

Western blot analysis was used to determine the protein expression levels of mesenchymal cell markers (Vimentin and $\mathrm{N}$-cadherin) and epithelial cell markers (E-cadherin and Keratin) in A549 cells. As shown in Fig. 4, the miR-145 mimic significantly increased the expression of E-cadherin and Keratin, and decreased the expression of Vimentin and $\mathrm{N}$-cadherin compared with that of the control group.
miR-145 restoration downregulates the expression of Oct4, Wnt $3 a$ and $\beta$-catenin in A549 cells. Following transfection of the miR-145 mimic into A548 cells, western blot analysis revealed that the relative protein levels of Oct4, Wnt3a and $\beta$-catenin were all significantly decreased in the miR-145 mimic group compared with those of the control group (Fig. 5).

Oct4 is a direct target of miR-145. Luciferase assays revealed that the translational activity of the luciferase-expressing plasmid containing the 3 'UTR of Oct4 was significantly 
supressed in the miR-145 mimic group compared with that of the control group (Fig. 6A).

Furthermore, in order to determine whether miR-145 affected the expression of Oct4, A549 cells were transfected with the miR-145 mimic and the miR-145 inhibitor. Western blot analysis revealed that Oct4 protein expression was significantly decreased in the miR-145 mimic group compared with that of the control group, while Oct 4 was significantly increased in the miR-145 inhibitor group (Fig. 6B).

Knockdown of Oct4 inhibits metastasis and EMT of A549 cells. In order to knockdown the expression of Oct4, small hairpin (sh) RNA-Oct4 was transfected into A549 cells. Western blot analysis revealed that the relative protein expression levels of Oct4 were significantly decreased in the shRNA-Oct4 group compared with those of the shRNA-NC group (Fig. 7).

Subsequently, the effect of Oct4 on metastasis and EMT in A549 cells was evaluated. Cell invasion assays revealed that following transfection with shRNA-Oct4, the number of invaded cells was significantly decreased compared with that of the control group $(21 \pm 3$ and $37 \pm 5$, respectively; $\mathrm{P}<0.05)$. In addition, cell adhesion assay revealed that the adhesion activity of A549 cells was markedly decreased in the group transfected with shRNA-Oct4 to $48.91 \%$ of the negative control group $(\mathrm{P}<0.01)$ (data not shown).

Furthermore, western blot analysis demonstrated that the expression of epithelial cell markers (E-cadherin and Keratin) were significantly increased in the shRNA-Oct4 group compared to that of the negative control group, whereas the expression of mesenchymal cell markers (Vimentin and $\mathrm{N}$-cadherin) was significantly decreased (Fig.8).

Knockdown of Oct4 downregulates the expression of Wnt3a and $\beta$-catenin in A549 cells. Western blot analysis was performed in order to examine the effects of Oct 4 on the protein expression levels of Wnt3a and $\beta$-catenin. As shown in Fig. 9, the expression levels of Wnt3a and $\beta$-catenin were significantly decreased in shRNA-Oct4-transfected cells compared with those of the shRNA-NC-transfected cells.

\section{Discussion}

Downregulation of miR-145 has been observed in various types of human cancer (11-13), and studies have indicated that the role of miR-145 in metastasis may be cell type-specific (14-16). The results of the present study revealed that miR-145 was downregulated in human lung cancer tissues as well as in a series of human lung cancer cell lines; this therefore indicated that miR-145 acted as an anti-oncogene in tumor development. Furthermore, an miR-145 mimic was transfected into A549 cells, which revealed that miR-145 restoration inhibited the invasive and adhesive abilities of A549 cells. These results therefore suggested that miR-145 had an inhibitory role in the regulation of lung cancer metastasis.

EMT is a process in which epithelial cells lose their morphology and gene expression patterns, including the molecular markers E-cadherin, $\alpha$-catenin and $\gamma$-catenin, as well as obtain mesenchymal cell morphology and gene expression characteristics, including the molecular markers $\mathrm{N}$-cadherin, Vimentin and $\alpha$-smooth muscle actin. EMT has been implicated in numerous pathological conditions. It exhibits a crucial role in promoting tumor progression and metastasis (18). Notably, EMT is reversible and mesenchymal-epithelial transition (MET) is the reverse process of EMT (19). MET is an important early event in somatic cell reprogramming (20). Therefore, studies have focused on elucidating the mechanisms in EMT regulation and identifying novel strategies for MET induction $(19,21)$. Certain miRNAs have been found to suppress EMT in cancer cells; however, the effect of miR-145 on EMT of lung cancer cells remains to be elucidated. The present study revealed that miR-145 restoration led to induced MET, as characterised by the altered expression of mesenchymal cell markers Vimentin and $\mathrm{N}$-cadherin, as well as epithelial cell markers E-cadherin and Keratin in lung cancer cells.

Furthermore, the present study aimed to investigate the mechanisms underlying the role of miR-145 in the regulation of EMT. Oct4 is an octamer motif-binding transcription factor that belongs to the Pit-Oct-Unc family (22). It was proposed that Oct4 acts as a multi-functional factor in cancer and stem cell biology; Oct4 is a key regulator that induces somatic cell pluripotency $(23,24)$. In addition, Oct4 has been shown to exhibit an oncogenic effect in several types of cancers (25-29). In the present study, luciferase assays and western blot analysis revealed that Oct4 was a directly downregulated by miR-145. Furthermore, the knockdown of Oct4 expression in lung cancer cells in the present study, significantly inhibited cell invasion and adhesion as well as induced MET. These results therefore indicated that miR-145 regulated EMT through targeting Oct4 in lung cancer cells.

The Wnt/ $\beta$-catenin signaling pathway has been reported to exhibit important roles in regulating the cellular processes involved in development, differentiation, proliferation and adult tissue homeostasis (30). Wnt $/ \beta$-catenin signaling is controlled at multiple levels; however, aberrant Wnt/ $\beta$-catenin signaling has been shown to be involved in the pathogenesis of multiple tumors and other disease states $(31,32)$. Previous studies have demonstrated that $\mathrm{Wnt} / \beta$-catenin signaling had important roles in the acquisition of an EMT phenotype and cancer metastasis (33-35). In addition Wnt/ $\beta$-catenin signaling was reported to be regulated by Oct4 in embryonic stem cells (36). The results of the present study demonstrated that following knockdown of Oct4 in lung cancer cells, Wnt3a and $\beta$-catenin expression was downregulated.

In conclusion, the present study demonstrated, for the first time, that miR-145 inhibited EMT in lung cancer cells through targeting the Oct4-mediated Wnt/ $\beta$-catenin signaling pathway. In view of the inhibitory role of miR-145 in lung cancer metastasis, these results may provide a potential novel approach for lung cancer therapy.

\section{References}

1. Jemal A, Siegel R, Xu J, et al: Cancer statistics, 2010. CA Cancer J Clin 60: 277-300, 2010.

2. Nguyen DX, Bos PD and Massagué J: Metastasis: from dissemination to organ-specific colonization. Nat Rev Cancer 9: 274-284, 2009.

3. Thiery JP: Epithelial-mesenchymal transitions in tumour progression. Nat Rev Cancer 2: 442-454, 2002.

4. Bates RC and Mercurio AM: The epithelial-mesenchymal transition (EMT) and colorectal cancer progression. Cancer Biol Ther 4: 365-370, 2005. 
5. Brabletz T, Hlubek F, Spaderna S, et al: Invasion and metastasis in colorectal cancer: epithelial-mesenchymal transition, mesenchymal-epithelial transition, stem cells and beta-catenin. Cells Tissues Organs 179: 56-65, 2005.

6. Kalluri R and Weinberg RA: The basics of epithelial-mesenchymal transition. J Clin Invest 119: 1420-1428, 2009.

7. Bracken CP, Gregory PA, Khew-Goodall Y and Goodall GJ: The role of microRNAs in metastasis and epithelial-mesenchymal transition. Cell Mol Life Sci 66: 1682-1699, 2009.

8. Bartel DP: MicroRNAs: genomics, biogenesis, mechanism, and function. Cell 116: 281-297, 2004.

9. Humphreys DT, Westman BJ, Martin DI and Preiss T: MicroRNAs control translation initiation by inhibiting eukaryotic initiation factor 4E/cap and poly(A) tail function. Proc Natl Acad Sci USA 102: 16961-16966, 2005.

10. Nicoloso MS, Spizzo R, Shimizu M, et al: MicroRNAs - the micro steering wheel of tumour metastases. Nat Rev Cancer 9 : 293-302, 2009.

11. Yanaihara N, Caplen N, Bowman E, et al: Unique microRNA molecular profiles in lung cancer diagnosis and prognosis. Cancer Cell 9: 189-198, 2006.

12. Sempere LF, Christensen M, Silahtaroglu A, et al: Altered microRNA expression confined to specific epithelial cell subpopulations in breast cancer. Cancer Res 67: 11612-11620, 2007.

13. Schepeler T, Reinert JT, Ostenfeld MS, et al: Diagnostic and prognostic microRNAs in stage II colon cancer. Cancer Res 68 6416-6424, 2008.

14. Sachdeva M and Mo YY: MicroRNA-145 suppresses cell invasion and metastasis by directly targeting mucin 1 . Cancer Res 70: 378-387, 2010.

15. Wang L, Tang H, Thayanithy V, et al: Gene networks and microRNAs implicated in aggressive prostate cancer. Cancer Res 69: 9490-9497, 2009.

16. Arndt GM, Dossey L, Cullen LM, et al: Characterization of global microRNA expression reveals oncogenic potential of miR-145 in metastatic colorectal cancer. BMC Cancer 9: 374, 2009.

17. Yin R, Zhang S, Wu Y, et al: microRNA-145 suppresses lung adenocarcinoma-initiating cell proliferation by targeting OCT4 Oncol Rep 25: 1747-1754, 2011.

18. Thiery JP, Acloque H, Huang RY, et al: Epithelial-mesenchymal transitions in development and disease. Cell 139: 871-890, 2009.

19. Lim J and Thiery JP: Epithelial-mesenchymal transitions: insights from development. Development 139: 3471-3486, 2012.

20. Samavarchi-Tehrani P, Golipour A, David L, et al: Functional genomics reveals a BMP-driven mesenchymal-to-epithelial transition in the initiation of somatic cell reprogramming. Cell Stem Cell 7: 64-77, 2010.
21. Zhang J and Ma L: MicroRNA control of epithelial-mesenchymal transition and metastasis. Cancer Metastasis Rev 31: 653-662, 2012.

22. Scholer H, Ruppert S, Suzuki N, Chowdhury K and Gruss P: New type of POU domain in germ line-specific protein Oct 4 . Nature 344:435-439, 1990.

23. Nichols J, Zevnik B, Anastassiadis K, et al: Formation of pluripotent stemcells in the mammalian embryo depends on the POU transcription factor Oct4. Cell 95: 379 391, 1998

24. de Jong J and Looijenga LH: Stem cell marker OCT3/4 in tumor biology and germ cell tumor diagnostics: history and future. Crit Rev Oncog 12: 171-203, 2006.

25. Kim RJ and Nam JS: OCT4 expression enhances features of cancer stem cells in a mouse model of breast cancer. Lab Anim Res 27: 147-152, 2011.

26. Zhang Y, Zhang X, Wang X, et al: Inhibition of LDH-A by lentivirus-mediated small interfering RNA suppresses intestinal-type gastric cancer tumorigenicity through the downregulation of Oct4. Cancer Lett 321: 45-54, 2012.

27. Chen Z, Xu WR, Qian H, et al: Oct4, a novel marker for human gastric cancer. J Surg Oncol 99: 414-419, 2009.

28. Guo Y, Liu S, Wang P, et al: Expression profile of embryonic stem cell-associated genes Oct4, Sox 2 and Nanog in human gliomas. Histopathology 59: 763-775, 2011.

29. Iida H, Suzuki M, Goitsuka R and Ueno H: Hypoxia induces CD133 expression in human lung cancer cells by up-regulation of OCT3/4 and SOX2. Int J Oncol 40: 71-79, 2012.

30. Chien AJ, Conrad WH and Moon RT: A Wnt survival guide: from flies to human disease. J Invest Dermatol 129: 1614-1627, 2009.

31. Chien AJ and Moon RT: WNTS and WNT receptors as therapeutic tools and targets in human disease processes. Front Biosci 12: 448-457, 2007.

32. Moon RT, Kohn AD, De Ferrari GV and Kaykas A: WNT and beta-catenin signalling: diseases and therapies. Nat Rev Genet 5: 691-701, 2004.

33. Fu Y, Zheng S, An N, et al: $\beta$-catenin as a potential key target for tumor suppression. Int J Cancer 129: 1541-1551, 2011.

34. Valenta T, Hausmann G and Basler K: The many faces and functions of $\beta$-catenin. EMBO J 31: 2714-2736, 2012.

35. Anson M, Crain-Denoyelle AM, Baud V, et al: Oncogenic $\beta$-catenin triggers an inflammatory response that determines the aggressiveness of hepatocellular carcinoma in mice. J Clin Invest 122: 586-599, 2012.

36. Davidson KC, Adams AM, Goodson JM, et al: Wnt/ $/$-catenin signaling promotes differentiation, not self-renewal, of human embryonic stem cells and is repressed by Oct 4 . Proc Natl Acad Sci USA 109: 4485-4490, 2012. 